\title{
Hemimetabolous genomes reveal molecular basis of termite eusociality
}

\author{
Mark C. Harrison ${ }^{1,12}$, Evelien Jongepier ${ }^{1,12}$, Hugh M. Robertson ${ }^{2,12}$, Nicolas Arning1, Tristan Bitard-Feildel', \\ Hsu Chao ${ }^{3}$, Christopher P. Childers ${ }^{4}{ }^{4}$, Huyen Dinh ${ }^{3}$, Harshavardhan Doddapaneni', Shannon Dugan ${ }^{3}$, \\ Johannes Gowin",6, Carolin Greiner,5, Yi Han ${ }^{3}$, Haofu Hu', Daniel S. T. Hughes ${ }^{3}$, Ann-Kathrin Huylmans $\mathbb{1}^{8}$, \\ Carsten Kemena', Lukas P. M. Kremer ${ }^{1}$ ', Sandra L. Lee ${ }^{3}$, Alberto Lopez-Ezquerra', Ludovic Mallet ${ }^{1}$, \\ Jose M. Monroy-Kuhn', Annabell Moser ${ }^{5}$, Shwetha C. Murali ${ }^{3}$, Donna M. Muzny ${ }^{3}$, Saria Otani7, \\ Maria-Dolors Piulachs ${ }^{9}$, Monica Poelchau ${ }^{4}$, Jiaxin $Q u^{3}$, Florentine Schaub ${ }^{5}$, Ayako Wada-Katsumata ${ }^{10}$, \\ Kim C. Worley ${ }^{3}$, Qiaolin Xie ${ }^{11}$, Guillem Ylla ${ }^{9}{ }^{9}$, Michael Poulsen $\mathbb{1}^{7}$, Richard A. Gibbs ${ }^{3}$, Coby Schal ${ }^{10}$, \\ Stephen Richards ${ }^{3}$, Xavier Belles ${ }^{9 *}{ }^{9 \star}$ Judith Korb $\mathbb{1}^{5,6 \star}$ and Erich Bornberg-Bauer ${ }^{1 \star}$
}

Around 150 million years ago, eusocial termites evolved from within the cockroaches, 50 million years before eusocial Hymenoptera, such as bees and ants, appeared. Here, we report the 2-Gb genome of the German cockroach, Blattella germanica, and the 1.3-Gb genome of the drywood termite Cryptotermes secundus. We show evolutionary signatures of termite eusociality by comparing the genomes and transcriptomes of three termites and the cockroach against the background of 16 other eusocial and non-eusocial insects. Dramatic adaptive changes in genes underlying the production and perception of pheromones confirm the importance of chemical communication in the termites. These are accompanied by major changes in gene regulation and the molecular evolution of caste determination. Many of these results parallel molecular mechanisms of eusocial evolution in Hymenoptera. However, the specific solutions are remarkably different, thus revealing a striking case of convergence in one of the major evolutionary transitions in biological complexity.

usociality, the reproductive division of labour with overlapping generations and cooperative brood care, is one of the major evolutionary transitions in biology ${ }^{1}$. Although rare, eusociality has been observed in a diverse range of organisms, including shrimps, mole rats and several insect lineages ${ }^{2-4}$. A particularly striking case of convergent evolution occurred within the holometabolous Hymenoptera and in the hemimetabolous termites (Isoptera), which are separated by over $350 \mathrm{Myr}$ of evolution ${ }^{5}$. Termites evolved within the cockroaches around 150 Myr ago, towards the end of the Jurassic period ${ }^{6,7}$, about 50 Myr before the first bees and ants appeared ${ }^{5}$. Therefore, identifying the molecular mechanisms common to both origins of eusociality is crucial to understanding the fundamental signatures of these rare evolutionary transitions. While the availability of genomes from many eusocial and non-eusocial hymenopteran species ${ }^{8}$ has allowed extensive research into the origins of eusociality within ants and bees ${ }^{9-11}$, a paucity of genomic data from cockroaches and termites has precluded large-scale investigations into the evolution of eusociality in this hemimetabolous clade.

The conditions under which eusociality arose differ greatly between the two groups. Termites and cockroaches are hemimetabolous and so show a direct development, while holometabolous hymenopterans complete the adult body plan during metamorphosis. In termites, workers are immatures and only reproductive castes are adults ${ }^{12}$, while in Hymenoptera, adult workers and queens represent the primary division of labour. Moreover, termites are diploid and their colonies consist of both male and female workers, and usually a queen and king dominate reproduction. This is in contrast to the haplodiploid system found in Hymenoptera, in which all workers and dominant reproductives are female. It is therefore intriguing that strong similarities have evolved convergently within the termites and the hymenopterans, such as differentiated castes and a nest life with reproductive division of labour. The termites can be subdivided into wood-dwelling and foraging termites. The former belong to the lower termites and produce simple, small colonies with totipotent workers that can become reproductives. Foraging termites (some lower and all higher termites) form large, complex societies, in which worker castes can be irreversible ${ }^{12}$. For this reason, higher, but not lower, termites can be classed as superorganismal ${ }^{13}$. Similarly, within Hymenoptera, varying levels of eusociality exist.

Here we provide insights into the molecular signatures of eusociality within the termites. We analysed the genomes of two lower and one higher termite species and compared them to the genome

IInstitute for Evolution and Biodiversity, University of Münster, Münster, Germany. ${ }^{2}$ Department of Entomology, University of Illinois at Urbana-Champaign, Urbana, IL, USA. ${ }^{3}$ Human Genome Sequencing Center, Department of Human and Molecular Genetics, Baylor College of Medicine, Houston, TX, USA. ${ }^{4}$ USDA-ARS, National Agricultural Library, Beltsville, MD, USA. ${ }^{5}$ Evolutionary Biology \& Ecology, University of Freiburg, Freiburg, Germany. ${ }^{6}$ Behavioral Biology, University of Osnabrück, Osnabrück, Germany. ${ }^{7}$ Ecology and Evolution, University of Copenhagen, Copenhagen, Denmark. ${ }^{8}$ nstitute of Science and Technology Austria, Klosterneuburg, Austria. ${ }^{9}$ Institut de Biologia Evolutiva, CSIC-University Pompeu Fabra, Barcelona, Spain. ${ }^{10}$ Department of Entomology and Plant Pathology, North Carolina State University, Raleigh, NC, USA. "China National GeneBank, Beijing Genomics Institute (BGI)-Shenzhen, Shenzhen, China. ${ }^{12}$ These authors contributed equally: Mark C. Harrison, Evelien Jongepier and Hugh M. Robertson.

*e-mail: xavier.belles@ibe.upf-csic.es; judith.korb@biologie.uni-freiburg.de; ebb@uni-muenster.de 
of the German cockroach, Blattella germanica, as a closely related non-eusocial outgroup. Furthermore, differences in expression between nymphs and adults of the cockroach were compared to differences in expression between workers and reproductives of the three termites, to gain insights into how expression patterns changed along with the evolution of castes. Using 15 additional insect genomes to infer background gene family turnover rates, we analysed the evolution of gene families along the transition from non-social cockroaches to eusociality in the termites. In this study, we concentrated particularly on two hallmarks of insect eusociality, as previously described for Hymenoptera, with the expectation that similar patterns occurred along with the emergence of termites. These are the evolution of a sophisticated chemical communication, which is essential for the functioning of a eusocial insect colony ${ }^{3,14,15}$, and major changes in gene regulation along with the evolution of castes $^{9,10}$. We also tested whether transposable elements spurred the evolution of gene families that were essential for the transition to eusociality.

\section{Evolution of genomes, proteomes and transcriptomes}

We sequenced and assembled the genome of the German cockroach, B. germanica (Ectobiidae), and of the lower, drywood termite Cryptotermes secundus (Kalotermitidae; for assembly statistics, see Supplementary Table 1$)$. The cockroach genome $(2.0 \mathrm{~Gb})$ is considerably larger than all three termite genomes. The genome size of C. secundus $(1.30 \mathrm{~Gb})$ is comparable to the higher, fungus-growing termite Macrotermes natalensis $\left(1.31 \mathrm{~Gb}\right.$, Termitidae) ${ }^{16}$, but more than twice as large as the lower, dampwood termite Zootermopsis nevadensis $\left(562 \mathrm{Mb}\right.$, Termopsidae) ${ }^{17}$. The smaller genomes of termites compared to the cockroach are in line with previous size estimations based on C-values ${ }^{18}$. The proteome of $B$. germanica $(29,216$ proteins) is also much larger than in the termites, where we find the proteome size in C. secundus $(18,162)$ to be similar to those of the other two termites (M. natalensis: 16,$140 ; Z$. nevadensis: 15,459; Fig. 1). In fact, the B. germanica proteome was the largest among all 21 arthropod species analysed here (Fig. 1). Strong evidential support for over $80 \%$ of these proteins in B. germanica (see Supplementary Information) and large expansions in many manually annotated gene families offer high confidence in the accuracy of this proteome size. We also compared gene expression between the four species. When comparing worker expression with queen expression in the termites and nymph expression (fifth and sixth instars) with adult female expression in B. germanica, we found shifts in specificity of expression for termites compared to the cockroach in several gene families (Fig. 2). It has previously been reported for the primitively eusocial paper wasp Polistes canadensis that the majority of caste-biased genes, especially those upregulated in workers, are novel genes ${ }^{19}$. The authors suggested that this may be a feature of early eusociality. We did not find the same pattern for the termites. Species-specific genes (those without an orthologue) were not enriched for differentially expressed genes in any of the termites, with slight peaks among Blattodea- and Isoptera-specific genes (Supplementary Fig. 1).

\section{Gene family expansions assisted by TEs in termites}

The transitions to eusociality in ants ${ }^{10}$ and bees ${ }^{9}$ have been linked to major changes in gene family sizes. Similarly, we detected significant gene family changes on the branch leading to the termites (seven expansions and ten contractions; Supplementary Fig. 2 and Supplementary Table 2). The numbers of species-specific, significant expansions and contractions of gene families varied within termites (Z.nevadensis: 15/5; C. secundus: 27/3; M. natalensis: 24/20; Supplementary Fig. 2 and Supplementary Tables 3-5). Interestingly, in B. germanica we measured 93 significant gene family expansions but no contractions (Supplementary Table 6), which contributed to the large proteome.
The termite and cockroach genomes contain a higher level of repetitive DNA compared to the hymenopterans we analysed (Fig. 1). C. secundus and B. germanica genomes both contain 55\% repetitive content (Supplementary Table 7), which is higher than in both Z.nevadensis (28\%) and thehighertermite M.natalensis (46\%; Fig. 1$)^{20}$. As also found in $Z$. nevadensis and M. natalensis ${ }^{20}$, LINEs and especially the subfamily BovB were the most abundant transposable elements (TEs) in the B. germanica and C. secundus genomes, indicating that a proliferation of LINEs may have occurred in the ancestors of Blattodea (cockroaches and termites).

We hypothesized that these high levels of TEs may be driving the high turnover in gene family sizes within the termites and $B$. germanica $^{21}$. Expanded gene families indeed had more repetitive content within $10-\mathrm{kb}$ flanking regions in all three termites $\left(P<1.3 \times 10^{-8}\right.$; Wald $t$-test; Supplementary Tables 8 and 9$)$, in particular in the higher termite M. natalensis. In contrast, gene family expansions were not correlated with TE content in flanking regions for B. germanica. These results suggest that a major expansion of LINEs at the root of the Blattodea clade contributed to the evolution of gene families within termites, probably via unequal crossingover $^{21}$; however, the expansions in B. germanica were not facilitated by TEs. It can therefore be speculated that the large expansion of LINEs within Blattodea allowed the evolution of gene families that ultimately facilitated the transition to eusociality.

\section{Expansion and positive selection of ionotropic receptors}

Insects perceive chemical cues from toxins, pathogens, food and pheromones with three major families of chemoreceptors, the odorant (ORs), gustatory and ionotropic (IRs) receptors ${ }^{22}$. ORs, in particular, have been linked to colony communication in eusocial Hymenoptera, where they abound ${ }^{14,15,23}$. Interestingly, as previously detected for $Z$. nevadensis ${ }^{17}$, the OR repertoire is substantially smaller in B. germanica and all three termites compared to hymenopterans. IRs, on the other hand, which are less frequent in hymenopterans, are strongly expanded in the cockroach and termite genomes (Fig. 3 and Supplementary Fig. 3). Intronless IRs, which are known to be particularly divergent ${ }^{24}$, show the greatest cockroach- and Blattodea-specific expansions (Fig. 3a, Blattodea-, Cockroach- and Group D-IRs). By far the most IRs among all investigated species were found in B. germanica (455 complete gene models), underlining that the capacity for detecting many different kinds of chemosensory cues is crucial for this generalist that thrives in challenging, human environments. In line with a specialization in diet and habitat, the total number of IRs is lower within the termites (Z. nevadensis: 141; C. secundus: 135; M. natalensis: 75). Nevertheless, IRs are more numerous in termites than in all other analysed species (except Nasonia vitripennis: 111). This is strikingly similar to the pattern for ORs in Hymenoptera, which are also highly numerous in non-eusocial outgroups as well as in eusocial sister species ${ }^{14,23,25}$.

We scanned each IR group for signs of species-specific positive selection. Within the Blattodea-specific intronless IRs, we found two codon positions under significant selection for the higher termite M. natalensis (codeml site models 7 and $8 ; P=5.4 \times 10^{-5}$ ). Within a subgroup of five sequences, this was more strongly pronounced with seven codon positions under significant positive selection for M. natalensis $\left(P<1.7 \times 10^{-10}\right)$. The positively evolving codons are situated within the two ligand-binding lobes of the receptors (Fig. $3 \mathrm{c}$ ), showing that a diversification of ligand specificity has occurred along with the transition to higher eusociality and a change from wood-feeding to fungus-farming in M. natalensis. Only two IRs were differentially expressed between nymphs and adult females in $B$. germanica. Underlining a change in expression along with the evolution of castes, we found 35 IRs to be differentially expressed between workers and queens in Z. nevadensis, 11 in C. secundus and 10 in M. natalensis (Fig. 2 and Supplementary Table 10). The possible 


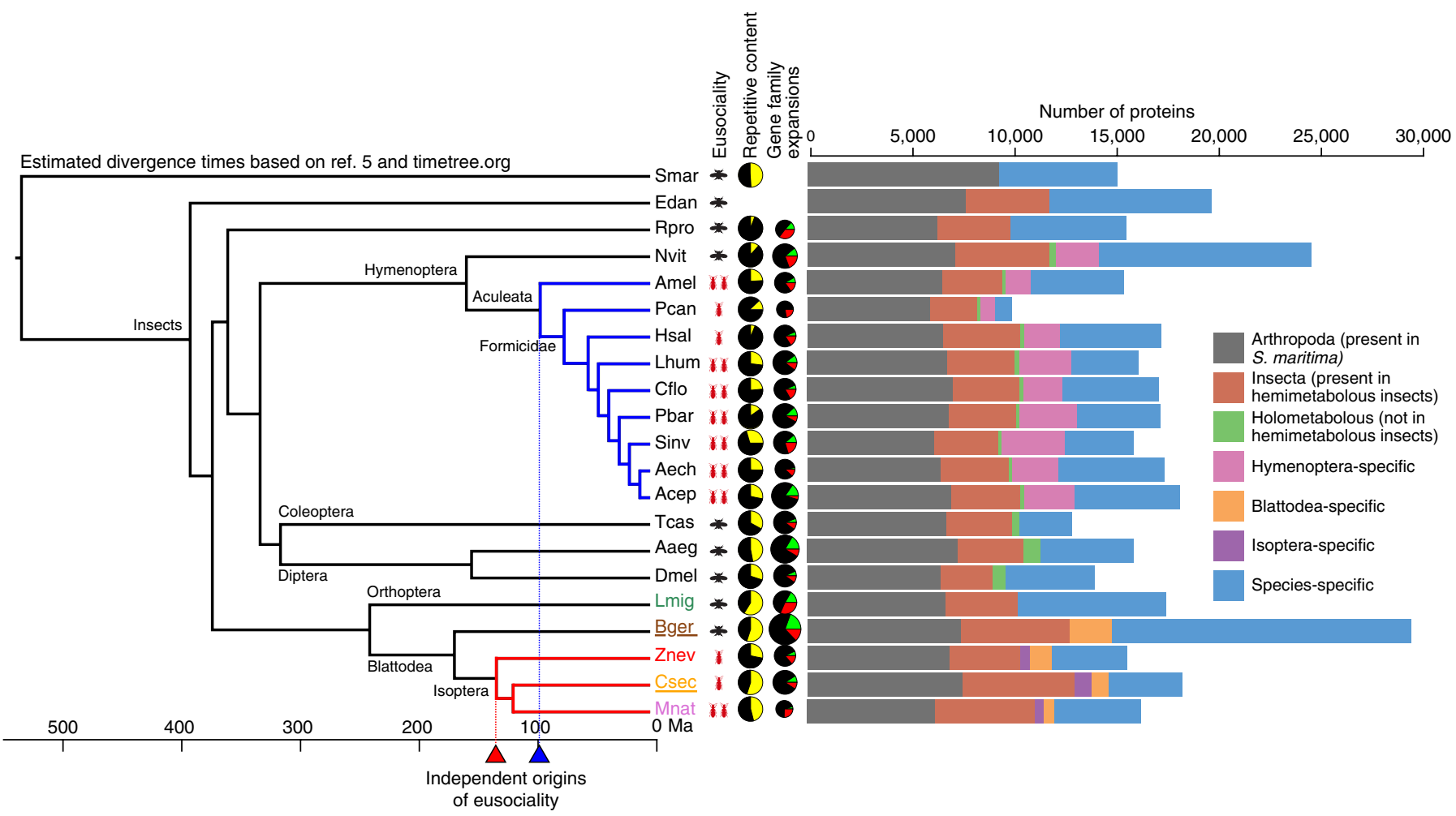

Fig. 1| Phylogenetic, genomic and proteomic comparisons of $\mathbf{2 0}$ insect species. From left to right: a phylogenetic tree of 20 insect species with Strigamia maritima (centipede) as the outgroup (species of newly sequenced genomes presented in this study are underlined); level of eusociality (one red insect: simple eusociality; two red insects: advanced eusociality; black fly: non-eusocial); fractions of repetitive content (yellow) within genomes of selected species (for sources, see Supplementary Information); proportions of species-specific gene family expansions (green), contractions (red) and stable gene families (black), the size of the pies represents the relative size of the gene family change (based on total numbers); a bar chart showing protein orthology across taxonomic groups within each genome. Ma, million years ago. Smar, Strigamia maritima; Edan, Ephemera danica; Rpro, Rhodnius prolixus; Nvit, Nasonia vitripennis; Amel, Apis mellifera; Pcan, Polistes canadensis; Hsal, Harpegnathos saltator; Lhum, Linepithema humile; Cflo, Camponotus floridanus; Pbar, Pogonomyrmex barbatus; Sinv, Solenopsis invicta; Aech, Acromyrmex echinatior; Acep, Atta cephalotes; Tcas, Tribolium castaneum; Aaeg, Aedes aegypti; Dmel, Drosophila melanogaster; Lmig, Locusta migratoria; Bger, Blattella germanica; Znev, Zootermopsis nevadensis; Csec, Cryptotermes secundus; Mnat, Macrotermes natalensis.

role of IRs in pheromonal communication has been highlighted both in the cockroach Periplaneta americana ${ }^{26}$ and in Drosophila melanogaster ${ }^{27}$, where several IRs show sex-biased expression.

One group of ORs (orange clade in Fig. 3b) is evolving under significant positive selection at codon positions within the second transmembrane domain in $M$. natalensis (codeml site model; $\left.P=1.1 \times 10^{-11}\right)$ and C. secundus $\left(P=5.6 \times 10^{-16}\right.$; Fig. $\left.3 \mathrm{~d}\right)$. Such a variation in the transmembrane domain can be related to ligand-binding specificity, as has been shown for a polymorphism in the third transmembrane domain for an OR in D. melanogaster ${ }^{28,29}$, adding further support for an adaptive evolution of chemoreceptors, in line with the greater need for a sophisticated colony communication in the termites. Similar to IRs, a higher proportion of ORs were differentially expressed between workers and queens in the three termites than between nymphs and adults in the cockroach (Fig. 2 and Supplementary Table 11), highlighting a change in expression and function along with the transition to eusociality. The evolution of chemoreceptors along with the emergence of the termites can also be related to adaptation processes and changes in diet compared to the cockroach. Experimental verification will help pinpoint which receptors are particularly important for communication.

\section{CHC-producing enzymes have evolved caste-specificity}

Despite their different ancestry, both termites and eusocial hymenopterans are characterized by the production of castespecific cuticular hydrocarbons (CHCs $)^{30-32}$, which are often crucial for regulating reproductive division of labour and chemical communication. Accordingly, we find changes in the termites in three groups of proteins involved in the synthesis of CHCs: desaturases (introduction of double bonds ${ }^{33}$ ), elongases (extension of C-chain length ${ }^{34}$ ) and CYP4G1 (last step of CHC biosynthesis ${ }^{35}$ ).

Desaturases are thought to be important for division of labour and social communication in ants ${ }^{36}$. As previously described for ants $^{36}$, Desat B genes are the most abundant desaturase family in the termites and the cockroach (Supplementary Table 12), especially in M. natalensis where we found ten gene copies (significant expansion; $P=0.0003$; Supplementary Table 5 and Supplementary Figure 4). As in ants, especially the first desaturases (Desat A-Desat E) vary greatly in their expression between castes and species in the three termites (Fig. 2 and Supplementary Table 13) ${ }^{36}$. In contrast to ants, where these genes are under strong purifying selection ${ }^{36}$, for the highly eusocial termite $M$. natalensis, we found significant positive selection within the Desat B genes (codeml site models 7 and $8 ; P=1.1 \times 10^{-16}$ ), indicating a diversification in function, possibly related to their greater diversification of worker castes (major and minor workers, major and minor soldiers). Although desaturases are often discussed in the context of CHC production and chemical communication, their biochemical roles are quite diverse $^{36}$, and the positive selection we observe for $M$. natalensis may, at least in part, be related to their rather different ecology of foraging and fungus-farming rather than nest-mate recognition. Future experimental verification of the function of these genes will help better understand these observed genomic and transcriptomic patterns. 

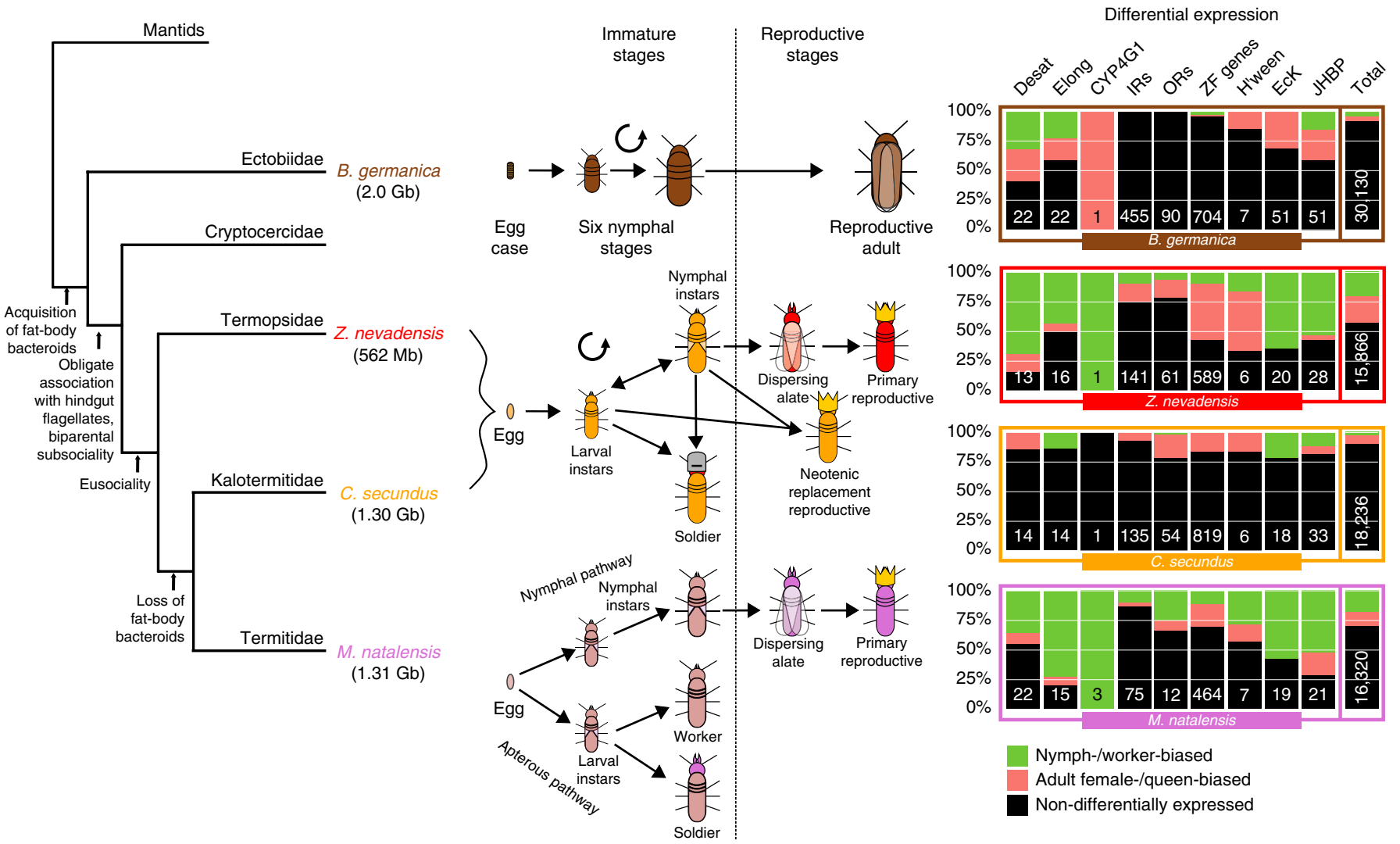

Fig. 2 | Comparison of developmental pathways between B. germanica, the lower termites Z. nevadensis and C. secundus, and the higher termite M. natalensis. Shown from left to right are: a simple phylogeny ${ }^{97}$ describing important novelties along the evolutionary trajectory to termites (numbers in brackets are genome sizes); life cycles; differential expression ( $\log _{2}$ (fold change) $>1$ and $P<0.05$; DESeq2 ${ }^{79}$; sample sizes are shown in the last column) between workers and queens (between nymphs and adult females in B. germanica) of the selected gene families (Desat, desaturases; Elong, elongases; H'ween, Halloween genes) and total numbers within all genes; the numbers denote total numbers of genes in each gene family.

Underlining an increased importance of $\mathrm{CHC}$ communication in termites, the expression patterns of elongases (extension of C-chain length) differ considerably in the termites compared to the cockroach (Fig. 2 and Supplementary Table 14). In contrast to B. germanica, in which elongases are both nymph- (five genes) and adult-biased (four genes), only one or two elongase genes in each termite are queen-biased in their expression, while many are workerbiased. As with the desaturases, a group of $M$. natalensis elongases also reveal significant signals of positive selection (codeml branchsite test; $P=4 \times 10^{-4}$ ), further indicating a greater diversification of $\mathrm{CHC}$ production in this higher termite.

The last step of CHC biosynthesis, the production of hydrocarbons from long-chain fatty aldehydes, is catalysed by a P450 gene, CYP4G1, in D. melanogaster ${ }^{35}$. We found one copy of CYP4G1 in B. germanica, Z. nevadensis and C. secundus, but three copies in $M$. natalensis, reinforcing the greater importance of $\mathrm{CHC}$ synthesis in this higher termite. Corroborating the known importance of maternal CHCs in B. germanica ${ }^{37}$, CYP4G1 is overexpressed in female adults compared to nymphs (Fig. 2 and Supplementary Table 15). In each of the termites, however, CYP4G1 is more highly expressed in workers (or kings in C. secundus) compared to queens (Fig. 2 and Supplementary Table 15), adding support that, compared to cockroach nymphs, a change in the dynamics and turnover of CHCs in termite workers has taken place.

\section{Changes in gene regulation in termites}

The development of distinct castes underlying division of labour is achieved via differential gene expression. Major changes in gene regulation have been reported as being central to the transition to eusociality in bees ${ }^{9}$ and ants ${ }^{10}$. Accordingly, we found major changes in putative DNA methylation patterns (levels per 1-to-1 orthologue) among the termites compared to four other hemimetabolous insect species (Fig. 4a). This is revealed by $\mathrm{CpG}$ depletion patterns $\left(\mathrm{CpG}_{\mathrm{o} / \mathrm{e}}\right.$, observed versus expected number of $\mathrm{CpGs)}$ ), a reliable predictor of DNA methylation ${ }^{38,39}$, correlating more strongly between the termites than among any of the other analysed hemimetabolous insects (Fig. 4). In other words, within orthologous genes, predicted DNA methylation levels differ greatly between termites and other hemimetabolous species but remain conserved among termite species.

The predicted levels of DNA methylation correlated negatively with the caste-specificity of expression for each of the termites. This is confirmed by a positive correlation between $\mathrm{CpG}_{\mathrm{o} / \mathrm{e}}$ (negative association with level of DNA methylation) and absolute $\log _{2}$ (fold change) of expression between queens and workers (Pearson's $r=0.32$ to $0.36 ; P<2.2 \times 10^{-16}$ ). The caste-specific expression of putatively unmethylated genes in termites is reflected in the enrichment of GO terms related to sensory perception, regulation of transcription, signalling and development, whereas methylated genes are mainly related to general metabolic processes (Fig. $4 \mathrm{~b}$ and Supplementary Table 16). These results show strong parallels to findings for eusocial Hymenoptera ${ }^{40-43}$. This is in stark contrast to the non-eusocial cockroach, B. germanica, where there was only a very weak relationship between $\mathrm{CpG}_{\mathrm{o} / \mathrm{e}}$ and differential expression between nymphs and adult females $(r=0.14)$, nor were any large differences apparent in enriched GO terms between putatively methylated and non-methylated genes (Fig. 4b).

Our results argue in favour of a diminished role of DNA methylation in caste-specific expression within eusocial insects, 


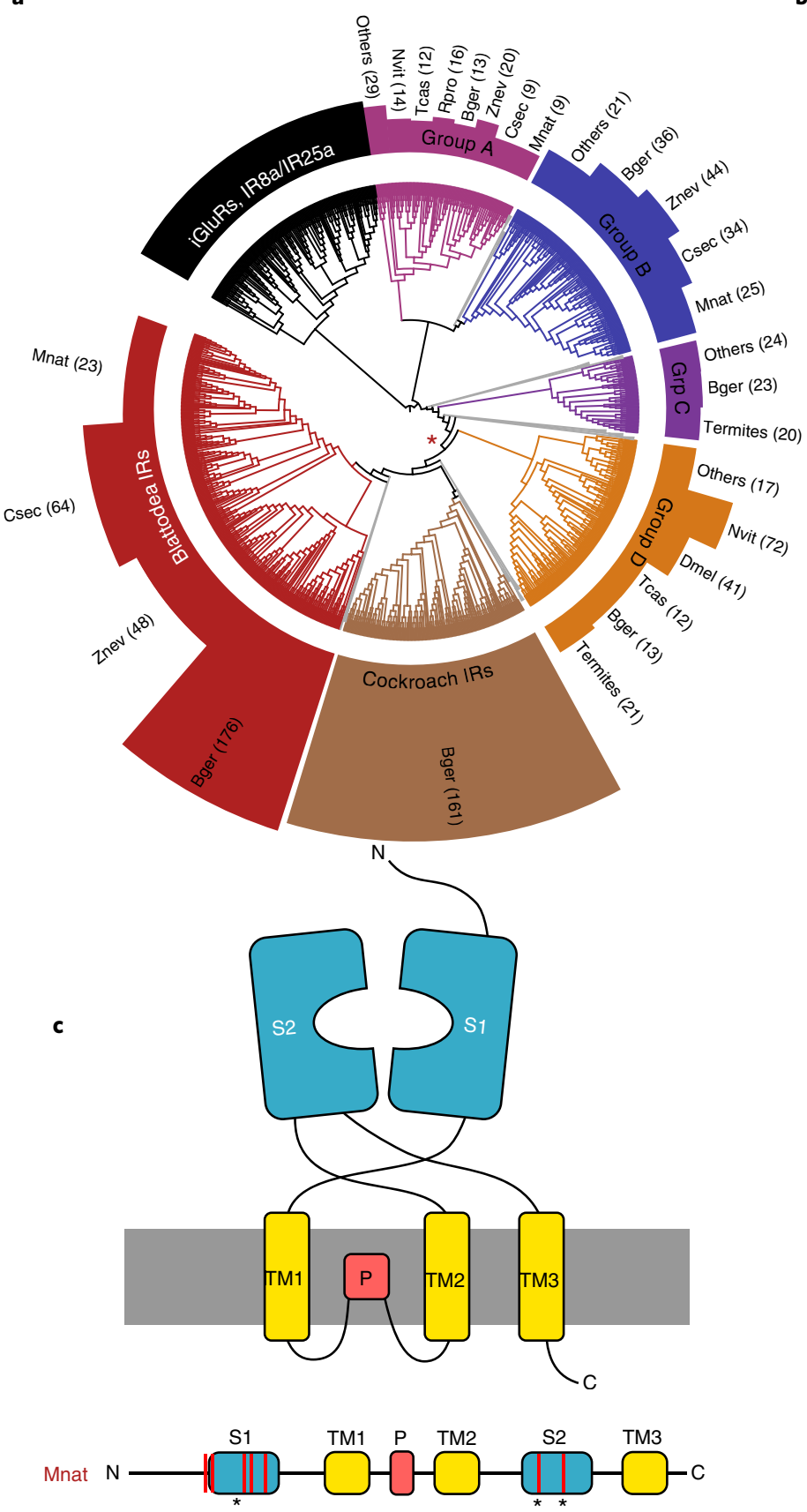

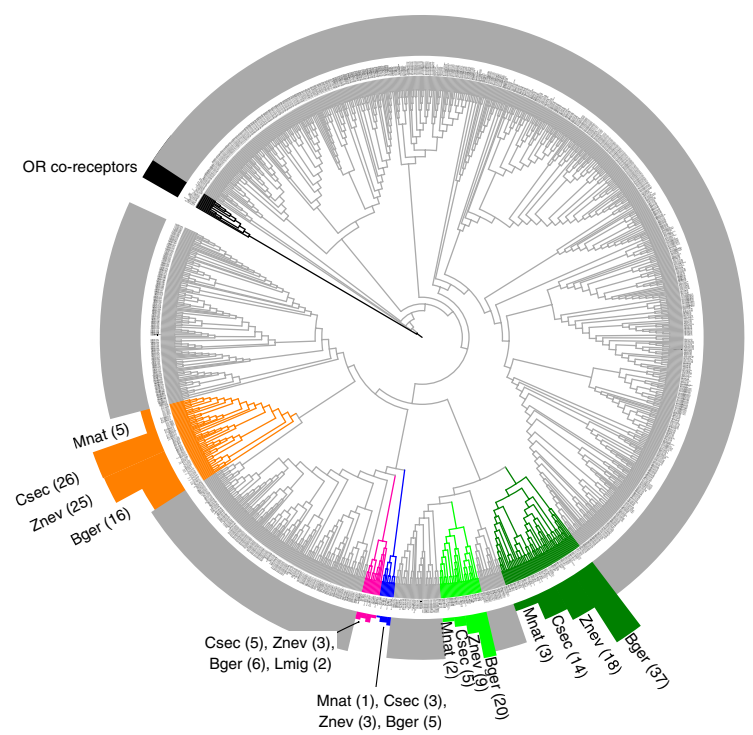

d

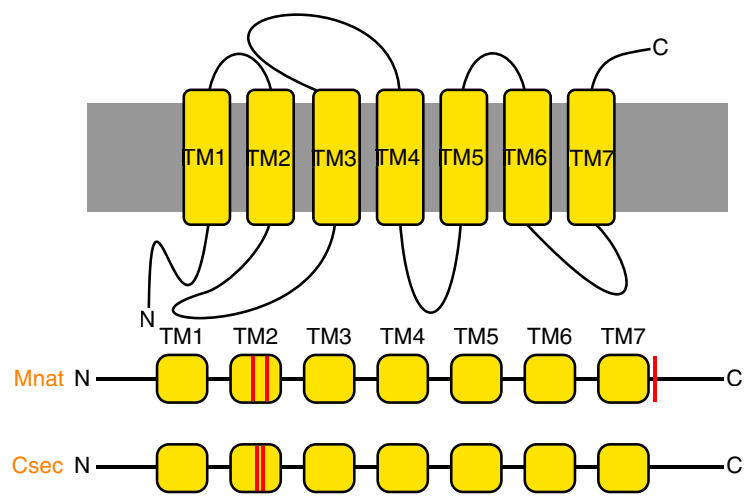

Fig. 3 Expansions, contractions and positive selection within IRs and ORs in termites. a,b, IR (a) and OR (b) gene trees of 13 insect species. In each tree, only well-supported clades (support values $>85$ ) that include B. germanica or termite genes are highlighted within the gene trees. The lengths of the coloured bars represent the number of genes per species within each of these clades. The red asterisk in a denotes the putative root of intronless IRs. c, The upper schematic diagram depicts the 2D structure of an IR, containing ligand-binding lobes (S1 and S2), transmembrane regions (TM1-3) and the pore domain $(P)$. Below, the sequence of the domains along the peptide is represented, showing that the sites, which are under significant positive selection (red bars; codeml site models 7 and 8 ) within Blattodea IRs for $M$. natalensis $\left(P<1.7 \times 10^{-10}\right.$; likelihood-ratio test, 5 sequences, 413 aligned codons), are all situated within the ligand-binding lobes and on or around the putative ligand-binding sites (asterisks) ${ }^{86}$. $\mathbf{d}$, The same representation for ORs, which include eight transmembrane regions. Positive selection was found for $M$. natalensis $\left(P=1.1 \times 10^{-10} ; 5\right.$ sequences, 1,001 aligned codons $)$ and C. secundus ( $P=5.6 \times 10^{-16}$; likelihood ratio test, 26 sequences, 1,913 aligned codons) of the orange clade, each at two codon positions within the second transmembrane region and at a third position within the carboxy-terminal extracellular region for $M$. natalensis.

as recently shown ${ }^{38,44}$. In fact, DNA methylation appears to be important for the regulation of housekeeping genes because predicted methylated genes are related to general biological processes (further supported by lower $\mathrm{CpG}_{\mathrm{o} / \mathrm{e}}$ within 1-to-1 orthologues than in non-conserved genes $)^{45}$, while caste-specific genes are 'released' from this type of gene regulation. However, a recent study linked caste-specific DNA methylation to alternative splicing in Z. nevadensis ${ }^{46}$. 
a

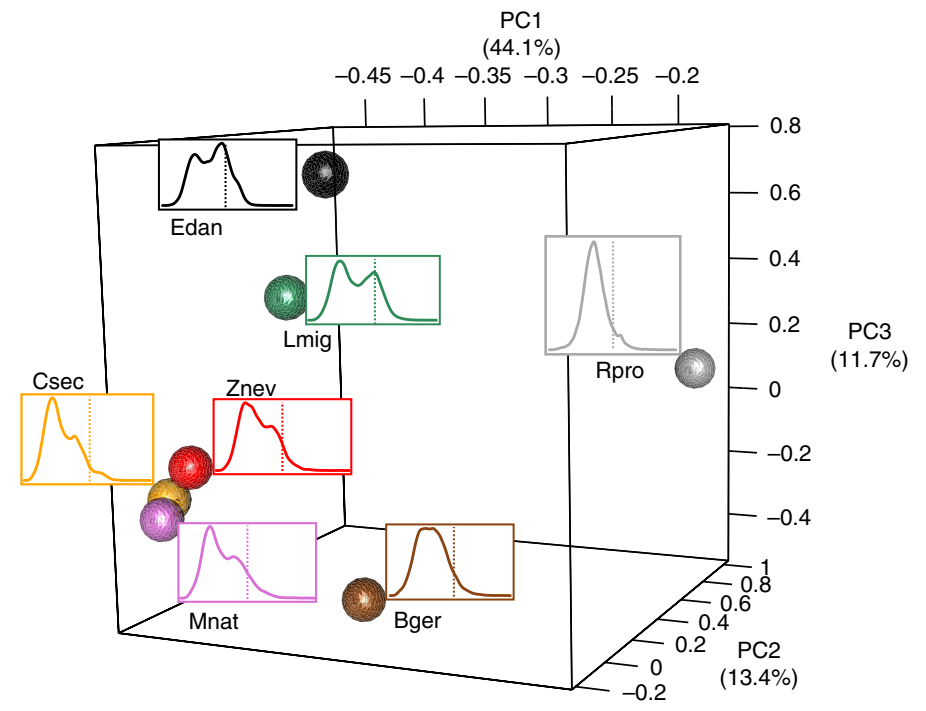

b
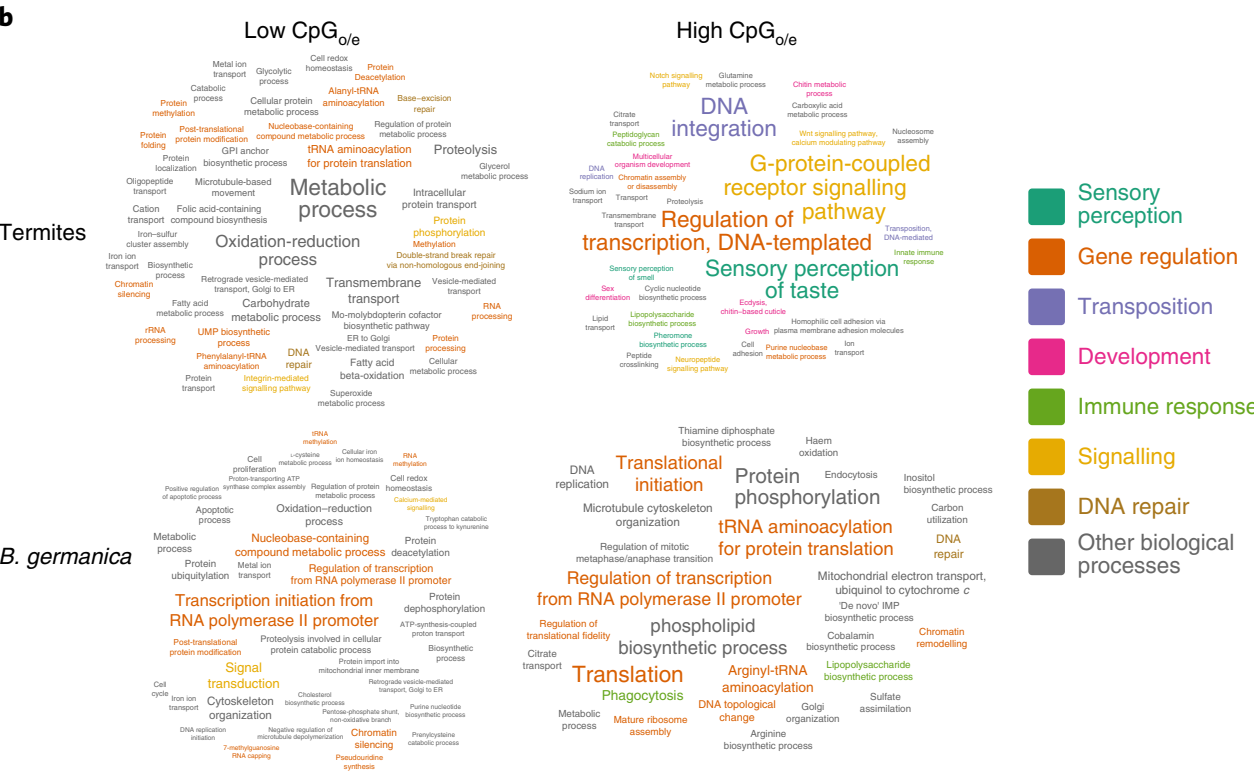

Fig. 4 | $\mathrm{CpG}_{\mathrm{o} / \mathrm{e}}$ of seven hemimetabolous insects. a, Principal component analysis (PCA) of predicted DNA methylation patterns among 2,664 1-to-1 orthologues, estimated via $\mathrm{CpG}_{0 / \mathrm{e}}$. The spheres represent the positions of the species within the 3D PCA, with the distance between the spheres representing the similarity of $\mathrm{CpG}_{0 / \mathrm{e}}$ between species at each orthologue; the curves are the distribution of $\mathrm{CpG}_{\mathrm{o} / \mathrm{e}}$, with the dotted line showing $\mathrm{CpG}$ o/e $=1$. b. Tag clouds of enriched $\left(P<0.05\right.$; Fisher test, weight algorithm, topGO $\left.{ }^{96}\right) \mathrm{GO}$ terms (biological processes) among the lower (left) and the higher quartile (right) of $\mathrm{CpG}_{\mathrm{o} / \mathrm{e}}$ within termites (top) and B. germanica (bottom). For termites, genes were merged from all three species for analysing $\mathrm{GO}$ term

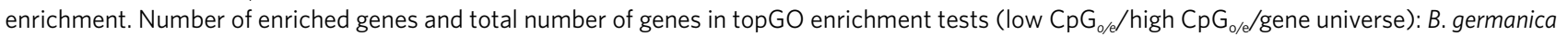
$(3,291 / 1,842 / 11,409) ;$ termites $(6,754 / 4,600 / 25,910)$. High $\mathrm{CpG}_{0 / \mathrm{e}}$ indicates a low level of DNA methylation and vice versa.

Major biological transitions are often accompanied by expansions of transcription factor (TF) families, such as genes containing zinc-finger (ZF) domains ${ }^{47}$. We also observed large differences in $\mathrm{ZF}$ families within the termites compared to B. germanica. Many ZF families were reduced or absent in termites, while different, unrelated ZF gene families were significantly expanded (Supplementary Tables 2-6). Queen-biased genes were significantly over-represented among ZF genes for each of the termites $\left(P<2 \times 10^{-10} ; \chi^{2}\right.$ test; Supplementary Table 17), indicating an important role of ZF genes in the regulation of genes related to caste-specific tasks and colony organization in the termites. This is in contrast to the significant under-representation of differentially expressed ZF genes within B. germanica $\left(P=4.8 \times 10^{-5} ; \chi^{2}\right.$-test $)$. Interestingly, two other important TF families (bHLH and bZIP) ${ }^{47}$, which were not expanded in the termites, showed no caste-specific expression pattern $(P>0.05)$, except bZIP genes, in which queen-biased genes were marginally over-represented for $M$. natalensis $(P=0.049)$. These major upheavals in ZF gene families and their caste-specific expression show that major changes in TFs accompanied the evolution of termites, strikingly similar to the evolution of ants ${ }^{10}$.

\section{Evolution of genes related to moulting and metamorphosis}

Hemimetabolous eusociality is characterized by differentiated castes, which represent different developmental stages. This is in contrast to eusocial Hymenoptera, in which workers and reproductives are adults. While cockroaches develop directly through several nymphal stages before becoming reproductive adults, termite development is more phenotypically plastic, and workers are essentially immatures (Fig. 2). In wood-dwelling termites, such as C. secundus and $Z$. nevadensis, worker castes are non-reproductive immatures 
that are totipotent to develop into other castes, while in the higher termite $M$. natalensis, workers can be irreversibly defined instars. It is therefore clear that a major change during the evolution of termites occurred within developmental pathways. Accordingly, we found changes in expression and gene family size of several genes related both to moulting and metamorphosis.

In the synthesis of the moulting hormone, 20-hydroxyecdysone, the six Halloween genes (five cytochrome P450s and a Rieske-domain oxygenase) play a key role ${ }^{48,49}$. Only one Halloween gene, Shade (Shd; CYP314A1), which mediates the final step of 20-hydroxyecdysone synthesis, is differentially expressed between the final nymphal stages and adult females in B. germanica (Fig. 2 and Supplementary Table 18), consistent with its role in the nymphal or imaginal moult. In the three termites, the Halloween genes show varying caste-specific expression (Fig. 2 and Supplementary Table 18), showing that ecdysone plays a significant role in the regulation of caste differences. Ecdysteroid kinase genes (EcK), which convert the insect moulting hormone into its inactive state, ecdysone 22-phosphate, for storage ${ }^{50}$, are only overexpressed in female adults compared to nymphs in B. germanica (16/51 genes, Fig. 2 and Supplementary Table 19). In termites, however, where the gene copy number is reduced (18 to 20 per species), these important moulting genes appear to have evolved worker-specific functions (Fig. 2 and Supplementary Table 19).

Whereas 20-hydroxyecdysone promotes moulting, juvenile hormone $(\mathrm{JH})$ represses imaginal development in pre-adult instars ${ }^{51}$. $\mathrm{JH}$ is important in caste differentiation in eusocial insects, including termites ${ }^{12,52}$. Haemolymph JH-binding proteins (JHBPs), which transport $\mathrm{JH}$ to its target tissues ${ }^{53}$, are reduced within the termites (21 to 33 genes) but significantly expanded in B. germanica (51 copies; $P=0018$; Supplementary Table 6). Thirteen of the JHBP genes are overexpressed in adult females and only 8 in nymphs in B. germanica (Fig. 2 and Supplementary Table 20). In both Z. nevadensis and $M$. natalensis, on the other hand, JHBPs are significantly more worker-biased ( $P<0.01, \chi^{2}$ test; Supplementary Table 20 and Fig. 2). In $C$. secundus, expression is more varied, with four workerbiased, seven king-biased and two queen-biased genes (Fig. 2 and Supplementary Table 20).

These changes in copy number and caste-specific expression of genes involved in moulting and metamorphosis within termites compared to the German cockroach demonstrate that changes occurred in the control of the developmental pathway along with the evolution of castes. However, this interpretation needs to be experimentally verified.

\section{Conclusions}

These results, considered alongside many studies on eusociality in Hymenoptera $^{9,10,14,36}$, provide evidence that major changes in gene regulation and the evolution of sophisticated chemical communication are fundamental to the transition to eusociality in insects. Strong changes in DNA methylation patterns correlated with broadscale modifications of expression patterns. Many of these modified expression patterns remained consistent among the three studied termite species and occurred within protein pathways essential for eusocial life, such as $\mathrm{CHC}$ production, chemoperception, ecdysteroid synthesis and $\mathrm{JH}$ transport. The stronger patterns we observe for M. natalensis, especially within genes linked to chemical communication, such as the expansion of Desat B and CYP4G1 genes and significant positive selection in desaturases, elongases and IRs, may be associated with this termite's higher level of eusociality and its status as a superoganism ${ }^{13}$. The analysis of further higher and lower termites would shed light on the generality of these patterns and possibly assist in the distinction between the influences of ecological and eusocial traits.

Many of the mechanisms implicated in the evolution of eusociality in the termites occurred convergently around 50 Myr later in the phylogenetically distant Hymenoptera. However, several details are unique due to the distinct conditions within which eusociality arose. One important difference is the higher TE content within cockroaches and termites, which probably facilitated changes in gene family sizes, supporting the transition to eusociality. However, the most striking difference is the apparent importance of IRs for chemical communication in the termites, compared to ORs in Hymenoptera. According to our results, the non-eusocial ancestors of termites possessed a broad repertoire of IRs, which favoured the evolution of important functions for colony communication in these chemoreceptors within the termites, whereas in the solitary ancestors of eusocial hymenopterans ORs were most abundant ${ }^{14,25}$. The parallel expansions of different chemoreceptor families in these two independent origins of eusociality indicate that convergent selection pressures existed during the evolution of colony communication in both lineages.

\section{Methods}

Genome sequencing and assembly. Genomic DNA from a single Blattella germanica male from an inbred line (strain: American Cyanamid $=$ Orlando Normal) was used to construct two paired-end (180-bp and 500-bp inserts) and one of the two mate-pair libraries (2-kb inserts). An 8-kb mate-pair library was constructed from a single female. The libraries were sequenced on an Illumina HiSeq2000 sequencing platform. The $413 \mathrm{~Gb}$ of raw sequence data were assembled with Allpaths LG $^{54}$, and then scaffolded and gap-filled using the in-house tools Atlas-Link v.1.0 (https://www.hgsc.bcm.edu/software/atlas-link) and Atlas gap-fill v.2.2. For Cryptotermes secundus, three paired-end libraries (250-bp, 500-bp and 800 -bp inserts) and three mate-pair libraries (2-kb, 5-kb and 10-kb inserts) were constructed from genomic DNA that was extracted from the head and thorax of 1,000 individuals, originating from a single, inbred field colony. The libraries were sequenced on an Illumina HiSeq2000 sequencing platform. The C. secundus genome was assembled using SOAPdenovo (v.2.04) ${ }^{55}$ with optimized parameters, followed by gapcloser (v1.10, released with SOAPdenovo) and kgf (v1.18, released with SOAPdenovo).

Transcriptome sequencing and assembly. For annotation purposes, 22 wholebody RNA-sequencing (RNA-Seq) samples from various developmental stages were obtained for B. germanica. For C. secundus, RNA-Seq libraries were obtained for three workers, four queens and four kings, based on degutted, whole-body extracts. In addition, we sequenced ten Macrotermes natalensis RNA-Seq libraries from three queens, one king and six pools of workers. All libraries were constructed using the Illumina (TruSeq) RNA-Seq kit.

For protein-coding gene annotation, B. germanica reads were assembled with de novo Trinity (version r2014-04-13) ${ }^{56}$. The C. secundus reads were assembled using Cufflinks on reads mapped with TopHat (version2.2.1 ${ }^{57,58}$, de novo Trinity ${ }^{56}$ and genome-guided Trinity on reads mapped with TopHat.

Repeat annotation. A custom C. secundus and B. germanica repeat library was constructed using a combination of homology-based and de novo approaches, including RepeatModeler/RepeatClassifier (http://www.repeatmasker.org/ RepeatModeler/), LTRharvest/LTRdigest ${ }^{59}$ and TransposonPSI (http:// transposonpsi.sourceforge.net/). The ab initio repeat library was complemented with the RepBase (update 29 August 2016) ${ }^{60}$ and SINE repeat databases, filtered for redundancy with CD-hit and classified with RepeatClassifier. RepeatMasker (version open-4.0.6, http://www.repeatmasker.org) was used to mask the C. secundus and B. germanica genome. Repeat content for the other studied species (Fig. 1) was obtained from the literature ${ }^{61-67}$.

Protein-coding gene annotation. The B. germanica genome was annotated with Maker (version 2.31 .8$)^{68}$, using the species-specific repeat library, B. germanica transcriptome data (22 whole-body RNA-Seq samples) and the Swiss-Prot/ UniProt database (last accessed: 21 January 2016) plus the C. secundus and Zootermopsis nevadensis protein sequences for evidence-based gene model predictions. AUGUSTUS (version 3.2) ${ }^{69}$, GeneMark-ES Suite (version 4.21) ${ }^{70}$ and SNAP $^{71}$ were used for ab initio predictions. C. secundus protein-coding genes were predicted using homology-based, ab initio and expression-based methods, and integrated into a final gene set (see Supplementary Information). Gene structures were predicted by GeneWise ${ }^{72}$. The ab initio annotations were predicted with AUGUSTUS $^{73}$ and SNAP ${ }^{71}$, retained if supported by both methods and integrated with the homology-based predictions using GLEAN ${ }^{74}$. Transcriptome-based gene models were merged with PASA ${ }^{75}$ and tested for coding potential with $\mathrm{CPC}^{76}$ and OrfPredictor ${ }^{77}$. PASA gene models were merged with the homology-based and $a b$ initio gene set, retaining the PASA models in case of overlap. Desaturases, elongases, chemosensory receptors, cytochrome P450s and genes involved in the juvenile hormone pathway were manually curated in Blattodea. 
Differential gene expression. The C. secundus and M. natalensis RNA-Seq libraries were complemented with nine published $Z$. nevadensis libraries, yielding two to six libraries from workers, queens and kings for each termite. These were compared to six of the B. germanica libraries: two from fifth instar nymphs, two from sixth instar nymphs and two from adult females. Reads were mapped to the genome using HiSat ${ }^{78}$. Read counts per gene were obtained using htseq-count and DESeq $2^{79}$ was used for differential expression analysis. Differential expression analysis between kings (males), queens (females) and workers (majors and minors combined for M. natalensis) was assessed for the termites. For $B$. germanica we evaluated the differential expression between adults and the two last nymphal stages combined, with the assumption that the final nymphal stages are homologous to termite workers and the adult females are homologous to termite queens. Genes were considered significantly differentially expressed if $P<0.05$ and $\log _{2}$ (fold change) $>|1|$ in order to account for allometric differences as recommended in a previous study ${ }^{80}$.

Protein orthology. In addition to B. germanica, C. secundus, Z. nevadensis and M. natalensis, 16 other insect proteomes were included in our analyses: Locusta migratoria, Rhodnius prolixus, Ephemera danica, Drosophila melanogaster, Aedes aegypti, Tribolium castaneum, Nasonia vitripennis, Polistes canadensis, Apis mellifera, Harpegnathos saltator, Linepithema humile, Camponotus floridanus, Pogonomyrmex barbatus, Solenopsis invicta, Acromyrmex echinatior and Atta cephalotes; as well as for the centipede Strigamia maritima as an outgroup (for sources, see Supplementary Table 22). These proteomes were grouped into orthologous clusters with OrthoMCL ${ }^{81}$, with a granularity of 1.5.

IR and OR identification, phylogeny and structure. Ionotropic receptors (IRs) were identified using two custom hidden Markov models (HMMs) obtained with hmmbuild and hmmpress of the HMMER suite ${ }^{82}$. The first HMM comprises the IR's ion channel and ligand-binding domain based on a MAFFT ${ }^{83}$ protein alignment of 76 IRs from 15 species (Supplementary Table 23). The second HMM was built to distinguish IRs from iGluRs, IR8a and IR25a, which have an additional amino-terminal domain ${ }^{24}$. For this we built an HMM from 48 protein sequences (Supplementary Table 23). The proteomes were scanned with pfam_scan and the two custom HMMs, where proteins that matched the IR HMM, but not the aminoterminal domain HMM were annotated as IRs. Odorant receptors (ORs) were identified on the basis of the Pfam domain PF02949 (7tm OR).

Multiple sequence alignments of IRs and ORs were obtained with hmmalign ${ }^{82}$, using the Pfam OR HMM PF02949 and custom IR HMM to guide the alignment. Gene trees were computed with FastTree ${ }^{84}$ (options: -pseudo -spr 4 -mlacc 2 -slownni) and visualized with iTOL v3 ${ }^{85}$. Putative IR ligand-binding residues and structural regions were identified on the basis of the alignments with D. melanogaster IRs and iGluRs of known structure ${ }^{86}$.

Gene family expansions and contractions. For the analyses of gene family expansions and contractions, the hierarchical clustering algorithm MC-UPGMA was used, with a ProtoLevel cutoff of $80\left(\right.$ ref. $\left.^{88}\right)$. Protein families were further divided into sub-families if they contained more than 100 proteins in a single species, or more than an average of 35 proteins per species. Proteins were blasted against the RepeatMasker TE database $\left(\mathrm{E}\right.$-value $\left.<10^{-5}\right)$ and clusters where $>50 \%$ of the proteins were identified as transposable elements were discarded. Cladeand species-specific protein family expansions and contractions, were identified with CAFE v3. $0^{89}$ using the same protocol as in previous studies ${ }^{9,10}$ (see also Supplementary Information).

TE-facilitated expansions. The repeat content in the $10-\mathrm{kb}$ flanking regions of B. germanica, C. secundus, Z. nevadensis and $M$. natalensis genes was calculated using bedtools ${ }^{90}$. Coding DNA sequences (CDSs) from neighbouring genes were removed and the repeat content was analysed using generalized linear mixed models (glmmPQL implemented in the $\mathrm{R}^{91}$ package $\mathrm{MASS}^{92}$ ) with binomial error distribution. Fixed predictors included gene family expansion, species ID and their interaction. Cluster ID was fitted as a random factor to avoid pseudo-replication. Significance was assessed on the basis of the Wald $t$-test $\left(\mathrm{R}\right.$ package aod $\left.{ }^{93}\right)$ at $\alpha<0.05$. Main and interaction effects for each of the genomic regions are listed in Supplementary Table 8. Model parameters are listed in Supplementary Table 8.

Tests for positive selection. To test for positive selection within gene families of interest, site model tests 7 and 8 were performed (model $=0$; NSsites $=78$ ) on species-specific CDS alignments, or branch-site test ( model $=2$; NSsites $=2$; fix omega $=1$ for null model and 0 for alternative model) on multi-species alignments. Protein sequences were aligned using MAFFT ${ }^{83}$ with the E-INS-i strategy, and CDS alignments were created using pal2nal.pl ${ }^{94}$. Phylogenetic trees were created with FastTree $^{84}$. Alignments were trimmed using Gblocks (settings: $-\mathrm{b} 2=21 ;-\mathrm{b} 3=20$; -b4 =5; -b5=a). Models were compared using likelihood-ratio test and where $P<0.05$, Bayes empirical Bayes results were consulted for codon positions under positive selection $(P<0.05)$.

CpG depletion patterns and GO enrichment. To estimate DNA methylation, we compared observed to expected CpG counts within CDS sequences ${ }^{38,39}$. A low
$\mathrm{CpG}_{\mathrm{o} / \mathrm{e}}$ indicates a high level of DNA methylation, as the cytosines of methylated $\mathrm{CpGs}$ often mutate to thymines. Expected $\mathrm{CpG}$ counts were calculated by dividing the product of cytosine and guanine counts by the sequence length. The principal component analysis in Fig. 4 was created using the R function prcomp on logtransformed $\mathrm{CpG}_{\mathrm{o} / \mathrm{e}}$ values for all 1-to-1 orthologues for the seven hemimetabolous species. These orthologues were extracted from the OrthoMCL results. The three-dimensional (3D) plot was created with the plot3d command from the $\mathrm{R}$ package rgl.

CpG-depleted (first quartile) and -enriched (fourth quartile) genes were tested for enrichment of Gene Ontology terms. Pfam protein domains were obtained for B. germanica, $Z$. nevadensis, $C$. secundus and $M$. natalensis protein sequences using PfamScan $^{95}$. Corresponding GO terms were obtained with Pfam2GO. GO-term over-representation was assessed using the TopGO ${ }^{96}$ package in R. Enrichment analysis was performed using the weight algorithm selecting nodesize $=10$ to remove terms with fewer than ten annotated GO terms. After that, GO terms classified as significant (topGOFisher $<0.05$ ) were visualized using the R package tagcloud (https://cran.r-project.org/web/packages/tagcloud/).

Life Science Reporting Summary. Further information on experimental design is available in the Life Sciences Reporting Summary.

Code availability. All custom-made scripts used in these analyses are available at the following repository: https://github.com/ebbgroup/Genomic-comparisons-inBlattodea.

Data availability. The genome assembly of Blattella germanica is archived on NCBI under the accession PRJNA203136. The genome assembly of Cryptotermes secundus is available on NCBI under the accession PRJNA381866. The additionally annotated genes for Z. nevadensis and $M$. natalensis are available from the Dryad Digital Repository: https://doi.org/10.5061/dryad.51d4r. Transcriptomic reads generated in this study are available in SRA (B. germanica: PRJNA382128; C. secundus: PRJNA382129; M. natalensis: PRJNA382034).

Received: 16 August 2017; Accepted: 19 December 2017; Published online: 5 February 2018

\section{References}

1. Szathmáry, E. \& Maynard Smith, J. The major evolutionary transitions. Nature 374, 227-232 (1995).

2. Andersson, M. The evolution of eusociality. Annu. Rev. Ecol. Syst. 15, 165-189 (1984)

3. Wilson, E. O. The Insect Societies (Harvard University Press, Cambridge, 1971).

4. Rubenstein, D. R. \& Abbot, P. (eds) Comparative Social Evolution (Cambridge University Press, Cambridge, 2017).

5. Misof, B. et al. Phylogenomics resolves the timing and pattern of insect evolution. Science 346, 763-767 (2014).

6. Legendre, F. et al. Phylogeny of Dictyoptera: dating the origin of cockroaches, praying mantises and termites with molecular data and controlled fossil evidence. PLoS One 10, e0130127 (2015).

7. Bourguignon, T. et al. The evolutionary history of termites as inferred from 66 mitochondrial genomes. Mol. Biol. Evol. 32, 406-421 (2015).

8. Elsner, D., Kremer, L. P., Arning, N. \& Bornberg-Bauer, E. Comparative genomic approaches to investigate molecular traits specific to social insects. Curr. Opin. Insect Sci. 16, 87-94 (2016).

9. Kapheim, K. M. et al. Genomic signatures of evolutionary transitions from solitary to group living. Science 348, 1139-1143 (2015).

10. Simola, D. F. et al. Social insect genomes exhibit dramatic evolution in gene composition and regulation while preserving regulatory features linked to sociality. Genome Res. 23, 1235-1247 (2013).

11. Woodard, S. H. et al. Genes involved in convergent evolution of eusociality in bees. Proc. Natl. Acad. Sci. USA 108, 7472-7477 (2011).

12. Korb, J. \& Hartfelder, K. Life history and development - a framework for understanding developmental plasticity in lower termites. Biol. Rev. 83, 295-313 (2008)

13. Boomsma, J. J. \& Gawne, R. Superorganismality and caste differentiation as points of no return: how the major evolutionary transitions were lost in translation. Biol. Rev. 93, 28-54 (2018).

14. Zhou, X. et al. Chemoreceptor evolution in Hymenoptera and its implications for the evolution of eusociality. Genome Biol. Evol. 7, 2407-2416 (2015).

15. Trible, W. et al. Orco mutagenesis causes loss of antennal lobe glomeruli and impaired social behavior in ants. Cell 170, 727-735.e10 (2017).

16. Poulsen, M. et al. Complementary symbiont contributions to plant decomposition in a fungus-farming termite. Proc. Natl Acad. Sci. USA 111, 14500-14505 (2014).

17. Terrapon, N. et al. Molecular traces of alternative social organization in a termite genome. Nat. Commun. 5, 3636 (2014).

18. Gregory, T. R. Animal Genome Size Database (accessed 25 November 2017); http://www.genomesize.com/. 
19. Ferreira, P. G. et al. Transcriptome analyses of primitively eusocial wasps reveal novel insights into the evolution of sociality and the origin of alternative phenotypes. Genome Biol. 14, R20 (2013).

20. Korb, J. et al. A genomic comparison of two termites with different social complexity. Front. Genet. 6, 9 (2015).

21. Kazazian, H. H. Mobile elements: drivers of genome evolution. Science 303, 1626-1632 (2004).

22. Joseph, R. M. \& Carlson, J. R. Drosophila chemoreceptors: a molecular interface between the chemical world and the brain. Trends Genet. 31, 683-695 (2015).

23. Brand, P. \& Ramírez, S. R. The evolutionary dynamics of the odorant receptor gene family in corbiculate bees. Genome Biol. Evol. 9, 2023-2036 (2017).

24. Croset, V. et al. Ancient protostome origin of chemosensory ionotropic glutamate receptors and the evolution of insect taste and olfaction. PLoS. Genet. 6, e1001064 (2010).

25. Robertson, H. M., Gadau, J. \& Wanner, K. W. The insect chemoreceptor superfamily of the parasitoid jewel wasp Nasonia vitripennis. Insect Mol. Biol. 19, 121-136 (2010).

26. Chen, Y., He, M., Li, Z.-Q., Zhang, Y.-N. \& He, P. Identification and tissue expression profile of genes from three chemoreceptor families in an urban pest, Periplaneta americana. Sci. Rep. 6, 27495 (2016).

27. Koh, T.-W. et al. The Drosophila IR20a clade of ionotropic receptors are candidate taste and pheromone receptors. Neuron 83, 850-865 (2014).

28. Pellegrino, M., Steinbach, N., Stensmyr, M. C., Hansson, B. S. \& Vosshall, L. B. A natural polymorphism alters odour and DEET sensitivity in an insect odorant receptor. Nature 478, 511-514 (2011).

29. Nichols, A. S. \& Luetje, C. W. Transmembrane segment 3 of Drosophila melanogaster odorant receptor subunit $85 \mathrm{~b}$ contributes to ligand-receptor interactions. J. Biol. Chem. 285, 11854-11862 (2010).

30. Oystaeyen, A. V. et al. Conserved class of queen pheromones stops social insect workers from reproducing. Science 343, 287-290 (2014).

31. Weil, T., Hoffmann, K., Kroiss, J., Strohm, E. \& Korb, J. Scent of a queencuticular hydrocarbons specific for female reproductives in lower termites. Naturwissenschaften 96, 315-319 (2009).

32. Dietemann, V., Peeters, C., Liebig, J., Thivet, V. \&Hölldobler, B. Cuticular hydrocarbons mediate discrimination of reproductives and nonreproductives in the ant Myrmecia gulosa. Proc. Natl Acad. Sci. USA 100, 10341-10346 (2003).

33. Dallerac, R. et al. A $\Delta 9$ desaturase gene with a different substrate specificity is responsible for the cuticular diene hydrocarbon polymorphism in Drosophila melanogaster. Proc. Natl Acad. Sci. USA 97, 9449-9454 (2000).

34. Finck, J., Berdan, E. L., Mayer, F., Ronacher, B. \& Geiselhardt, S. Divergence of cuticular hydrocarbons in two sympatric grasshopper species and the evolution of fatty acid synthases and elongases across insects. Sci. Rep. 6, 33695 (2016).

35. Qiu, Y. et al. An insect-specific P450 oxidative decarbonylase for cuticular hydrocarbon biosynthesis. Proc. Natl. Acad. Sci. USA 109, 14858-14863 (2012)

36. Helmkampf, M., Cash, E. \& Gadau, J. Evolution of the insect desaturase gene family with an emphasis on social Hymenoptera. Mol. Biol. Evol. 32, 456-471 (2015).

37. Fan, Y., Eliyahu, D. \& Schal, C. Cuticular hydrocarbons as maternal provisions in embryos and nymphs of the cockroach Blattella germanica. J. Exp. Biol. 211, 548-554 (2008).

38. Bewick, A. J., Vogel, K. J., Moore, A. J. \& Schmitz, R. J. Evolution of DNA methylation across insects. Mol. Biol. Evol. 34, 654-655 (2017).

39. Park, J. et al. Comparative analyses of DNA methylation and sequence evolution using Nasonia genomes. Mol. Biol. Evol. 28, 3345-3354 (2011).

40. Elango, N., Hunt, B. G., Goodisman, M. A. D. \& Yi, S. V. DNA methylation is widespread and associated with differential gene expression in castes of the honeybee, Apis mellifera. Proc. Natl. Acad. Sci. USA 106, 11206-11211 (2009).

41. Standage, D. S. et al. Genome, transcriptome and methylome sequencing of a primitively eusocial wasp reveal a greatly reduced DNA methylation system in a social insect. Mol. Ecol. 25, 1769-1784 (2016).

42. Patalano, S. et al. Molecular signatures of plastic phenotypes in two eusocial insect species with simple societies. Proc. Natl. Acad. Sci. USA 112, 13970-13975 (2015).

43. Rehan, S. M., Glastad, K. M., Lawson, S. P. \& Hunt, B. G. The genome and methylome of a subsocial small carpenter bee, Ceratina calcarata. Genome Biol. Evol. 8, 1401-1410 (2016)

44. Libbrecht, R., Oxley, P. R., Keller, L. \& Kronauer, D. J. C. Robust DNA methylation in the clonal raider ant brain. Curr. Biol. 26, 391-395 (2016).

45. Foret, S., Kucharski, R., Pittelkow, Y., Lockett, G. A. \& Maleszka, R. Epigenetic regulation of the honey bee transcriptome: unravelling the nature of methylated genes. BMC Genom. 10, 472 (2009).

46. Glastad, K. M., Gokhale, K., Liebig, J. \& Goodisman, M. A. D. The caste- and sex-specific DNA methylome of the termite Zootermopsis nevadensis. Sci. Rep. 6, 37110 (2016).
47. Schmitz, J. F., Zimmer, F. \& Bornberg-Bauer, E. Mechanisms of transcription factor evolution in Metazoa. Nucleic Acids Res. 44, 6287-6297 (2016).

48. Rewitz, K. F., Rybczynski, R., Warren, J. T. \& Gilbert, L. I. The Halloween genes code for cytochrome P450 enzymes mediating synthesis of the insect moulting hormone. Biochem. Soc. Trans. 34, 1256-1260 (2006).

49. Lang, M. et al. Mutations in the neverland gene turned Drosophila pachea into an obligate specialist species. Science 337, 1658-1661 (2012).

50. Sonobe, H. et al. Purification, kinetic characterization, and molecular cloning of a novel enzyme, ecdysteroid 22-kinase. J. Biol. Chem. 281, 29513-29524 (2006).

51. Jindra, M., Belles, X. \& Shinoda, T. Molecular basis of juvenile hormone signaling. Curr. Opin. Insect Sci. 11, 39-46 (2015).

52. Korb, J. in Genomics, Physiology and Behaviour of Social Insects Vol. 48 (eds Zayed, A. \& Kent, C. F.) 131-161 (Academic Press, 2015).

53. Kolodziejczyk, R. et al. Insect juvenile hormone binding protein shows ancestral fold present in human lipid-binding proteins. J. Mol. Biol. 377, 870-881 (2008).

54. Gnerre, S. et al. High-quality draft assemblies of mammalian genomes from massively parallel sequence data. Proc. Natl. Acad. Sci. USA 108, 1513-1518 (2011).

55. Li, Y., Hu, Y., Bolund, L. \& Wang, J. State of the art de novo assembly of human genomes from massively parallel sequencing data. Hum. Genomics 4, 271-277 (2010).

56. Grabherr, M. G. et al. Full-length transcriptome assembly from RNA-Seq data without a reference genome. Nat. Biotechnol. 29, 644-652 (2011).

57. Kim, D. et al. TopHat2: accurate alignment of transcriptomes in the presence of insertions, deletions and gene fusions. Genome Biol. 14, R36 (2013).

58. Roberts, A., Trapnell, C., Donaghey, J., Rinn, J. L. \& Pachter, L. Improving RNA-Seq expression estimates by correcting for fragment bias. Genome Biol. 12, R22 (2011).

59. Ellinghaus, D., Kurtz, S. \& Willhoeft, U. LTRharvest, an efficient and flexible software for de novo detection of LTR retrotransposons. BMC Bioinforma. 9, 18 (2008)

60. Bao, W., Kojima, K. K. \& Kohany, O. Repbase Update, a database of repetitive elements in eukaryotic genomes. Mob. DNA 6, 11 (2015).

61. Chipman, A. D. et al. The first myriapod genome sequence reveals conservative arthropod gene content and genome organisation in the centipede Strigamia maritima. PLoS Biol. 12, e1002005 (2014).

62. Mesquita, R. D. et al. Genome of Rhodnius prolixus, an insect vector of Chagas disease, reveals unique adaptations to hematophagy and parasite infection. Proc. Natl. Acad. Sci. USA 112, 14936-14941 (2015).

63. Nene, V. et al. Genome sequence of Aedes aegypti, a major arbovirus vector Science 316, 1718-1723 (2007).

64. The Honeybee Genome Sequencing Consortium. Insights into social insects from the genome of the honeybee Apis mellifera. Nature 443, 931-949 (2006).

65. Gadau, J. et al. The genomic impact of 100 million years of social evolution in seven ant species. Trends Genet.. 28, 14-21 (2012).

66. Richards, S. et al. The genome of the model beetle and pest Tribolium castaneum. Nature 452, 949-955 (2008).

67. Wang, X. et al. The locust genome provides insight into swarm formation and long-distance flight. Nat. Commun. 5, 2957 (2014).

68. Holt, C. \& Yandell, M. MAKER2: an annotation pipeline and genomedatabase management tool for second-generation genome projects. BMC Bioinforma. 12, 491 (2011).

69. Keller, O., Kollmar, M., Stanke, M. \& Waack, S. A novel hybrid gene prediction method employing protein multiple sequence alignments. Bioinformatics 27, 757-763 (2011).

70. Lomsadze, A., Ter-Hovhannisyan, V., Chernoff, Y. O. \& Borodovsky, M. Gene identification in novel eukaryotic genomes by self-training algorithm. Nucleic Acids Res. 33, 6494-6506 (2005).

71. Korf, I. Gene finding in novel genomes. BMC Bioinforma. 5, 59 (2004).

72. Birney, E., Clamp, M. \& Durbin, R. GeneWise and Genomewise. Genome Res 14, 988-995 (2004).

73. Stanke, M. et al. AUGUSTUS: ab initio prediction of alternative transcripts. Nucleic Acids Res. 34, W435-W439 (2006).

74. Elsik, C. G. et al. Creating a honey bee consensus gene set. Genome Biol. 8, R13 (2007).

75. Campbell, M. A., Haas, B. J., Hamilton, J. P., Mount, S. M. \& Buell, C. R. Comprehensive analysis of alternative splicing in rice and comparative analyses with Arabidopsis. BMC Genomics 7, 327 (2006).

76. Kong, L. et al. CPC: assess the protein-coding potential of transcripts using sequence features and support vector machine. Nucleic Acids Res. 35 , W345-W349 (2007)

77. Min, X. J., Butler, G., Storms, R. \& Tsang, A. OrfPredictor: predicting protein-coding regions in EST-derived sequences. Nucleic Acids Res. 33 W677-W680 (2005)

78. Pertea, M., Kim, D., Pertea, G. M., Leek, J. T. \& Salzberg, S. L. Transcriptlevel expression analysis of RNA-seq experiments with HISAT, StringTie and Ballgown. Nat. Protoc. 11, 1650-1667 (2016). 
79. Love, M. I., Huber, W. \& Anders, S. Moderated estimation of fold change and dispersion for RNA-seq data with DESeq2. Genome Biol. 15, 550 (2014).

80. Montgomery, S. H. \& Mank, J. E. Inferring regulatory change from gene expression: the confounding effects of tissue scaling. Mol. Ecol. 25, 5114-5128 (2016).

81. Li, L., Stoeckert, C. J. \& Roos, D. S. OrthoMCL: identification of ortholog groups for eukaryotic genomes. Genome Res. 13, 2178-2189 (2003).

82. Eddy, S. R. Profile hidden Markov models. Bioinformatics 14, 755-763 (1998).

83. Katoh, K. \& Standley, D. M. MAFFT multiple sequence alignment software version 7: improvements in performance and usability. Mol. Biol. Evol. 30 , 772-780 (2013).

84. Price, M. N., Dehal, P. S. \& Arkin, A. P. FastTree: computing large minimum evolution trees with profiles instead of a distance matrix. Mol. Biol. Evol. 26, 1641-1650 (2009).

85. Letunic, I. \& Bork, P. Interactive tree of life (iTOL) v3: an online tool for the display and annotation of phylogenetic and other trees. Nucleic Acids Res. 44 W242-W245 (2016).

86. Benton, R., Vannice, K. S., Gomez-Diaz, C. \& Vosshall, L. B. Variant ionotropic glutamate receptors as chemosensory receptors in Drosophila. Cell 136, 149-162 (2009)

87. Loewenstein, Y., Portugaly, E., Fromer, M. \& Linial, M. Efficient algorithms for accurate hierarchical clustering of huge datasets: tackling the entire protein space. Bioinformatics 24, i41-i49 (2008).

88. Rappoport, N., Linial, N. \& Linial, M. ProtoNet: charting the expanding universe of protein sequences. Nat. Biotechnol. 31, 290-292 (2013).

89. Han, M. V., Thomas, G. W. C., Lugo-Martinez, J. \& Hahn, M. W. Estimating gene gain and loss rates in the presence of error in genome assembly and annotation using CAFE 3. Mol. Biol. Evol. 30, 1987-1997 (2013).

90. Quinlan, A. R. \& Hall, I. M. BEDTools: a flexible suite of utilities for comparing genomic features. Bioinformatics 26, 841-842 (2010).

91. R Core Team. R: A Language and Environment for Statistical Computing (R Foundation for Statistical Computing, Vienna, 2014).

92. Venables, W. \& Ripley, B. Modern Applied Statistics with S, 4th edn (Springer, New York, 2002)

93. Lesnoff, M. \& Lancelot, R. aod: Analysis of Overdispersed Data R Package Version 1.3 (2012); http://cran.r-project.org/package=aod

94. Suyama, M., Torrents, D. \& Bork, P. PAL2nal: robust conversion of protein sequence alignments into the corresponding codon alignments. Nucleic Acids Res. 34, W609-W612 (2006).

95. Finn, R. D. et al. The Pfam protein families database: towards a more sustainable future. Nucleic Acids Res. 44, D279-D285 (2016).

96. Alexa, A. \& Rahnenführer, J. topGO: Enrichment Analysis for Gene Ontology R package version 2.30 .0 (2016); http://bioconductor.org/packages/release/ bioc/html/topGO.html

97. Bell, W. J., Roth, L. M. \& Nalepa, C. A. Cockroaches: Ecology, Behavior, and Natural History (JHU Press, Baltimore, 2007).

\section{Acknowledgements}

We thank O. Niehuis for allowing use of the unpublished E. danica genome, J. Gadau and C. Smith for comments and advice on the manuscript, and J. Schmitz for assistance with analyses and proofreading the manuscript. J.K. thanks Charles Darwin University (Australia), especially S. Garnett and the Horticulture and Aquaculture team, for providing logistic support to collect C. secundus. The Parks and Wildlife Commission, Northern Territory, the Department of the Environment, Water, Heritage and the Arts gave permission to collect (Permit number 36401) and export (Permit WT2010-6997) the termites. USDA is an equal opportunity provider and employer. M.C.H. and E.J. are supported by DFG grant BO2544/11-1 to E.B.-B. J.K. is supported by University of Osnabrück and DFG grant KO1895/16-1. X.B. and M.-D.P. are supported by Spanish
Ministerio de Economía y Competitividad (CGL2012-36251 and CGL2015-64727-P to X.B., and CGL2016-76011-R to M.-D.P.), including FEDER funds, and by Catalan Government (2014 SGR 619). C.S. is supported by grants from the US Department of Housing and Urban Development (NCHHU-0017-13), the National Science Foundation (IOS-1557864), the Alfred P. Sloan Foundation (2013-5-35 MBE), the National Institute of Environmental Health Sciences (P30ES025128) to the Center for Human Health and the Environment, and the Blanton J. Whitmire Endowment. M.P. is supported by a Villum Kann Rasmussen Young Investigator Fellowship (VKR10101).

\section{Author contributions}

E.B.-B. conceived, managed and coordinated the project; M.C.H., E.J. and H.M.R. are joint first authors. J.K. conceived and managed the $C$. secundus sequencing project, and coordinated termite-related analyses; S.R. conceived and managed the B. germanica sequencing project; S.R., S.D., S.L.L., H.C., H.V.D., H.D., Y.H., J.Q., S.C.M., D.S.T.H., K.C.W., D.M.M. and R.A.G. carried out B. germanica library construction, genome sequencing and assembly; C.S. and A.W-.K. provided biological material through full-sib mating for B. germanica; X.B. and C.S. co-managed the B. germanica analysis; M.P. and C.P.C. implemented Web Apollo data traces; S.O. and M.P. provided biological material for M. natalensis; C.G., J.G., J.M.M.-K., A.M., F.S., H.H. and J.K. coordinated and carried out DNA and RNA sequencing for C. secundus; M-D.P., X.B. and G.Y. coordinated transcriptome sequencing of B. germanica; L.M. performed automated gene prediction on C. secundus; E.J. and N.A. improved assembly and annotation for B. germanica \& $C$. secundus, and compared and analysed genome sizes and quality. E.J., N.A. and L.P.M.K. analysed TEs; M.C.H. analysed CpG patterns and signatures of selection; T.B-F., E.J., C.K., L.P.M.K. and A.L-E. performed orthology and phylogenetic analyses; L.P.M.K., E.J., H.M.R. and M.C.H. analysed gene family evolution; A.L-E., E.J. and M.C.H. analysed transcriptomes and performed differential expression analyses; T.B.-F. and A.L-E. carried out orthoMCL clustering; H.M.R. corrected gene models for chemoreceptors; C.K. and E.J. corrected gene models for desaturases and elongases; A-K.H. and M.C.H. corrected gene models for cytochrome P450s; E.B.-B. and M.C.H drafted and wrote the manuscript; X.B., M.-D.P. and J.K. contributed to sections of the manuscript; E.J. L.P.M.K., A.L-E., C.K. and M.C.H. wrote and organized the Supplementary Information L.P.M.K., N.A., A.L-E., M.C.H. and E.B.-B. prepared figures for the manuscript. All authors read, corrected and commented on the manuscript.

\section{Competing interests}

The authors declare no competing financial interests.

\section{Additional information}

Supplementary information is available for this paper at https://doi.org/10.1038/ s41559-017-0459-1.

Reprints and permissions information is available at www.nature.com/reprints. Correspondence and requests for materials should be addressed to X.B. or J.K. or E.B.-B. Publisher's note: Springer Nature remains neutral with regard to jurisdictional claims in published maps and institutional affiliations.

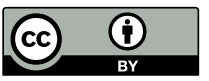

Open Access This article is licensed under a Creative Commons Attribution 4.0 International License, which permits use, sharing, adaptation, distribution and reproduction in any medium or format, as long as you give appropriate credit to the original author(s) and the source, provide a link to the Creative Commons license, and indicate if changes were made. The images or other third party material in this article are included in the article's Creative Commons license, unless indicated otherwise in a credit line to the material. If material is not included in the article's Creative Commons license and your intended use is not permitted by statutory regulation or exceeds the permitted use, you will need to obtain permission directly from the copyright holder. To view a copy of this license, visit http:// creativecommons.org/licenses/by/4.0/. 


\section{natureresearch}

Corresponding author(s): Mark C. Harrison

Initial submission $\square$ Revised version $\quad$ Final submission

\section{Life Sciences Reporting Summary}

Nature Research wishes to improve the reproducibility of the work that we publish. This form is intended for publication with all accepted life science papers and provides structure for consistency and transparency in reporting. Every life science submission will use this form; some list items might not apply to an individual manuscript, but all fields must be completed for clarity.

For further information on the points included in this form, see Reporting Life Sciences Research. For further information on Nature Research policies, including our data availability policy, see Authors \& Referees and the Editorial Policy Checklist.

\section{- Experimental design}

\section{Sample size}

Describe how sample size was determined.

2. Data exclusions

Describe any data exclusions.

3. Replication

Describe whether the experimental findings were reliably reproduced.

\section{Randomization}

Describe how samples/organisms/participants were allocated into experimental groups.

5. Blinding

Describe whether the investigators were blinded to group allocation during data collection and/or analysis.
For our differential expression analyses the sample size is predetermined by the number of genes, since we were comparing full transcriptomes between conditions.

For the kings of the termite Macrotermes natalensis, the sequencing of several samples failed, leading to only two replicates. We therefore did not conduct or report the results of any statistical tests with these samples.

For the differential expression analyses we only reported results for which at least 3 replicates were available. For DESeq2, the package with which we calculated differential expression, it is standard practice to work with 3 or more replicates.

This is not relevant. The experimental groups were determined by the caste membership of an individual.

not relevant

Note: all studies involving animals and/or human research participants must disclose whether blinding and randomization were used.

\section{Statistical parameters}

For all figures and tables that use statistical methods, confirm that the following items are present in relevant figure legends (or in the Methods section if additional space is needed).

n/a $\mid$ Confirmed

\The exact sample size $(n)$ for each experimental group/condition, given as a discrete number and unit of measurement (animals, litters, cultures, etc.)

$\triangle$ A description of how samples were collected, noting whether measurements were taken from distinct samples or whether the same sample was measured repeatedly

\ A statement indicating how many times each experiment was replicated

$\square$ The statistical test(s) used and whether they are one- or two-sided (note: only common tests should be described solely by name; more complex techniques should be described in the Methods section)

$\bigotimes$ A description of any assumptions or corrections, such as an adjustment for multiple comparisons

$\square$ \ The test results (e.g. $P$ values) given as exact values whenever possible and with confidence intervals noted

Х $\square$ A clear description of statistics including central tendency (e.g. median, mean) and variation (e.g. standard deviation, interquartile range) $\bigotimes \square$ Clearly defined error bars 
Policy information about availability of computer code

\section{Software}

Describe the software used to analyze the data in this study.
The software used is described in detail within the methods and the supplementary material. These are:

Genome assembly: Allpaths LG, SOAPdenovo, gapcloser, kgf

Transcriptome assembly: Trinity, Cufflinks, TopHat

Repeat annotations: RepeatModeler/RepeatClassifier, LTRharvest/LTRdigest,

TransposonPSI, CD-hit, Repeat Classifier, RepeatMasker

Annotation: Maker, AUGUSTUS, GeneMark-ES Suite, SNAP, GeneWise, PASA,

GLEAN, CPC, OrfPredictor

Differential gene expression: HiSat2, DESeq2

Protein orthology: OrthoMCL

IR and OR identification: HMMER suite, MAFFT

Gene family expansions and contractions: MC-UPGMA, CAFE

Test for positive selection: codeml of the PAML suite

GO enrichment: pfam2GO, topGO.

Many custom-made scripts available on request.

For manuscripts utilizing custom algorithms or software that are central to the paper but not yet described in the published literature, software must be made available to editors and reviewers upon request. We strongly encourage code deposition in a community repository (e.g. GitHub). Nature Methods guidance for providing algorithms and software for publication provides further information on this topic.

\section{- Materials and reagents}

Policy information about availability of materials

\section{Materials availability}

Indicate whether there are restrictions on availability of unique materials or if these materials are only available for distribution by a for-profit company.

\section{Antibodies}

Describe the antibodies used and how they were validated for use in the system under study (i.e. assay and species).

10. Eukaryotic cell lines

a. State the source of each eukaryotic cell line used.

b. Describe the method of cell line authentication used.

c. Report whether the cell lines were tested for mycoplasma contamination.

d. If any of the cell lines used are listed in the database of commonly misidentified cell lines maintained by ICLAC, provide a scientific rationale for their use. no unique materials

\section{not applicable}

$\mathrm{n} / \mathrm{a}$

$\mathrm{n} / \mathrm{a}$

$\mathrm{n} / \mathrm{a}$

$\mathrm{n} / \mathrm{a}$

\section{- Animals and human research participants}

Policy information about studies involving animals; when reporting animal research, follow the ARRIVE guidelines

\section{Description of research animals}

Provide details on animals and/or animal-derived materials used in the study. worker, queen and kings of the two termite species: Cryptotermes secundus and Macrotermes natalensis

Nymphs (5th and 6th instars) and adult females of Blattella germanica

Policy information about studies involving human research participants

\section{Description of human research participants}

Describe the covariate-relevant population characteristics of the human research participants. 